\title{
Are patients willing to accept longer travel times to decrease their risk associated with surgical procedures? A systematic review
}

\author{
Stefanie Bühn ${ }^{1 *}$, Jakob Holstiege ${ }^{2}$ and Dawid Pieper ${ }^{1}$
}

\begin{abstract}
Background: Distance to a hospital is an influencing factor for patients' decision making when choosing a hospital for surgery. It is unclear whether patients prefer to travel further to regional instead of local hospitals if the risk associated with elective surgery is lower in the farther hospital. The aim of our systematic review was to investigate patient preferences for the location of care, taking into consideration surgical outcomes and hospital distance.

Methods: MEDLINE (PubMed), EconLit, Psyclnfo and EMBASE were searched until November 2019. We included experimental choice studies in which participants were asked to make a hypothetical decision where to go for elective surgery when surgical risk and/or distance to the hospitals vary. There was no restriction on the type of intervention or study. Reviewers independently extracted data using a standardized form. The number and proportion of participants willing to accept additional risk to obtain surgery in the local hospital was recorded. We also extracted factors associated with the decision.

Results: Five studies exploring participants' preferences for local care were included. In all studies, there were participants who, independently of a decreased mortality risk or a higher survival benefit in the regional hospital, adhered to the local hospital. The majority of the patients were willing to travel longer to lower their surgical risk. Older age and fewer years of formal education were associated with a higher risk tolerance in the local hospital.

Conclusions: Whether patients were willing to travel for a lower surgery-associated risk could not be answered in a straightforward manner. The studies we identified showed that decision making also relies on factors other than on rational information on risk or distance to hospital.
\end{abstract}

Trial registration: International prospective register of ongoing systematic reviews (PROSPERO): CRD42016033655. Registered 1 January 2016.

\section{Background}

Studies show that patients who make an active decision rarely rely only on a completely rational decisionmaking process but rather use other than rational comparative information. Patients' active decision making is described as an active investigation and acquisition of information to make a conscious decision on healthrelated issues [1]. A scoping review shows that patients' decision-making process is complex and heterogeneous [1]. Patients' decisions often rely on their previous

\footnotetext{
* Correspondence: Stefanie.Buehn@uni-wh.de

${ }^{1}$ Institute for Research in Operative Medicine, Faculty of Health, School of Medicine, University Witten/Herdecke, Ostmerheimer Str. 200, Building 38, D-51109 Cologne, Germany

Full list of author information is available at the end of the article
}

experiences in a hospital or on social influences such as recommendations by friends. Another influencing factor when choosing a hospital is accessibility. Patients prefer a health care provider that is nearby, so that travel time will be short. Younger age, higher education and willingness to travel in general are factors associated with more tolerance toward greater distances to a hospital. Some studies indicate that the distance to a hospital is even more important than medical outcomes [1-3]. Besides surgical reputation and surgical competency, hospital reputation and distance to the hospital are the primarily important attributes by which patients choose their surgeon [4]. In another study, previous personal experiences in the hospital were the most frequently stated criterion,

(c) The Author(s). 2020 Open Access This article is distributed under the terms of the Creative Commons Attribution 4.0 International License (http://creativecommons.org/licenses/by/4.0/), which permits unrestricted use, distribution, and 
followed by the hospital's reputation, recommendation from one's own outpatient caregivers, distance from home and recommendations from relatives [5].

In past years, numerous studies pointed out that in surgical disciplines in particular, there is a positive relationship between high-volume hospitals and outcomes, especially for mortality $[6,7]$. To improve patients' surgical outcomes, policy makers introduced minimum hospital volumes. Consequently, centralisation of these procedures occurs. Besides positive effects for the patients' health on the one hand, centralisation of care to highvolume hospitals may lead to longer travel distances and increased travel burden. Patients might prefer local care for different reasons, for example, because it has advantages in terms of proximity to supportive family members and other local personal support systems, and it offers the possibility to receive a continuity of care $[8,9]$.

Distance to a hospital has a great impact on patients' decision making, as shown by numerous studies $[1-4,10,11]$. Our main research aim is to investigate whether and to what extent patients consider surgical risk and travel distances in their decision-making process when choosing a hospital for elective surgery.

With our systematic review, we aim to identify choice experimental studies investigating patient preferences for the location of a hospital for elective surgery, taking into consideration surgery-associated outcomes and hospital distance. Our study should contribute to the discussion about the need to include patients' preferences and values when making health care decisions about centralisation of clinical care and minimum surgery volumes.

\section{Methods}

\section{Protocol and registration}

This systematic review was registered with the international prospective register of systematic reviews (PROSPERO) (https://www.crd.york.ac.uk/PROSPERO/ display_record.php?RecordID=33655).

\section{Eligibility criteria}

We included experimental choice studies in which some kind of choice behavior measurement (trade-off, standard gamble, etc.) was performed. Participants needed to make a hypothetical decision on where to go for elective surgery when surgery-associated risk and/or distance to the hospitals (in terms of travel time or distance) vary. There was a restriction neither on the type of intervention nor on the medical discipline. There was no restriction on eligible underlying study types. Letters, editorials, and comments were excluded.

\section{Information sources}

A systematic literature search in the MEDLINE (through PubMed), EconLit, PsycInfo and EMBASE bibliographic databases was performed from inception until November 2019 (DP, SB). References to the included studies were retrieved and assessed for relevance. Corresponding authors of the included studies were contacted to ask whether they knew any unpublished, recently published or ongoing studies that could be relevant to our review (SB). Our search strategy included search terms regarding distance and travel connected with patient preferences and choice experiments connected with search terms regarding risks and outcomes in the surgical field. The full electronic search strategy of MEDLINE is presented in Additional file 1. Search terms were adapted for every database searched.

\section{Study selection}

Titles and abstracts were screened independently by two members $(\mathrm{DP} / \mathrm{JH}$ or $\mathrm{DP} / \mathrm{SB})$ of the research team. The full text of potentially eligible articles was retrieved, and two reviewers (DP, SB) independently assessed the eligibility of full texts against the review inclusion criteria. Any disagreement was resolved by discussion. When no agreement could be reached, a third member of the team was asked for final judgment. Data were extracted by one reviewer (SB) into piloted, structured summary tables and checked for accuracy and completeness by a second reviewer $(\mathrm{JH})$. Any disagreement was resolved when consensus was reached.

\section{Data collection process}

For each study, the main characteristics including country, the kind of scenario and methods to elicit preferences were extracted. Information about the setting, inclusion and exclusion criteria and travel time to the hospital were also extracted. As outcome, the number and proportion of participants willing to accept additional risk to obtain surgery in the local hospital was recorded. We also extracted factors associated with the decision.

\section{Risk of bias of individual studies}

To judge the risk of bias (RoB) in the included studies, we referred to the Grading of Recommendations, Assessment, Development and Evaluation (GRADE) publication on assessing certainty of evidence in the importance of outcomes and patients' preferences [12]. Domains in which the RoB should be assessed are: [1] selection of participants into the study, [2] completeness of data, [3] measurement instrument and [4] data analysis. We omitted the second domain (completeness of data) because in the identified studies, the no-response rate and the loss to follow-up were not involved. The quality of the included studies was rated by two reviewers (SB, DP) independently, and consensus was reached by discussion. 


\section{Results}

\section{Study selection}

After screening 3553 titles and abstracts, 16 full-text publications were included for a detailed evaluation. Five studies met the inclusion criteria. The detailed study selection process is provided in Fig. 1. A list of the excluded studies with reasons is provided in Additional file 2.

\section{Study characteristics}

We found three studies performed in the United States [8, 13, 14], one in Canada [9] and one in Germany [15]. All studies used hypothetical scenarios. In the study by Finlayson et al., the clinic's inpatients and outpatients who were awaiting another kind of elective surgery were asked to imagine that they had potentially resectable pancreas cancer [8]. In the study by Landau et al., patients who were diagnosed with an asymptomatic abdominal aortic aneurysm (AAA), but had no planned surgery in the immediate future, were to imagine they had to undergo surgery for their AAA [9]. The third study, by Chang et al., included parents with children who were referred to hospital because of suspected heart abnormalities, but finally none were diagnosed which subsequently were not confirmed by diagnosis [13]. They were told to imagine that their children had to undergo open-heart surgery. Afterward, they were asked to imagine the situation for themselves. In the fourth study, patients who presented themselves for evaluation of a pelvic mass but with no proved cancer diagnosis, were asked to imagine that they were diagnosed with ovarian cancer and scheduled for surgery [14]. In the study by Burkamp et al., participants were asked to imagine that they had to make a decision on which hospital to choose for elective total knee arthroplasty [15].

Characteristics of choice experiments of individual studies To elicit patients' preferences, all studies used discrete choice experiments (DCE). Patients were initially informed about the disease scenarios they should imagine

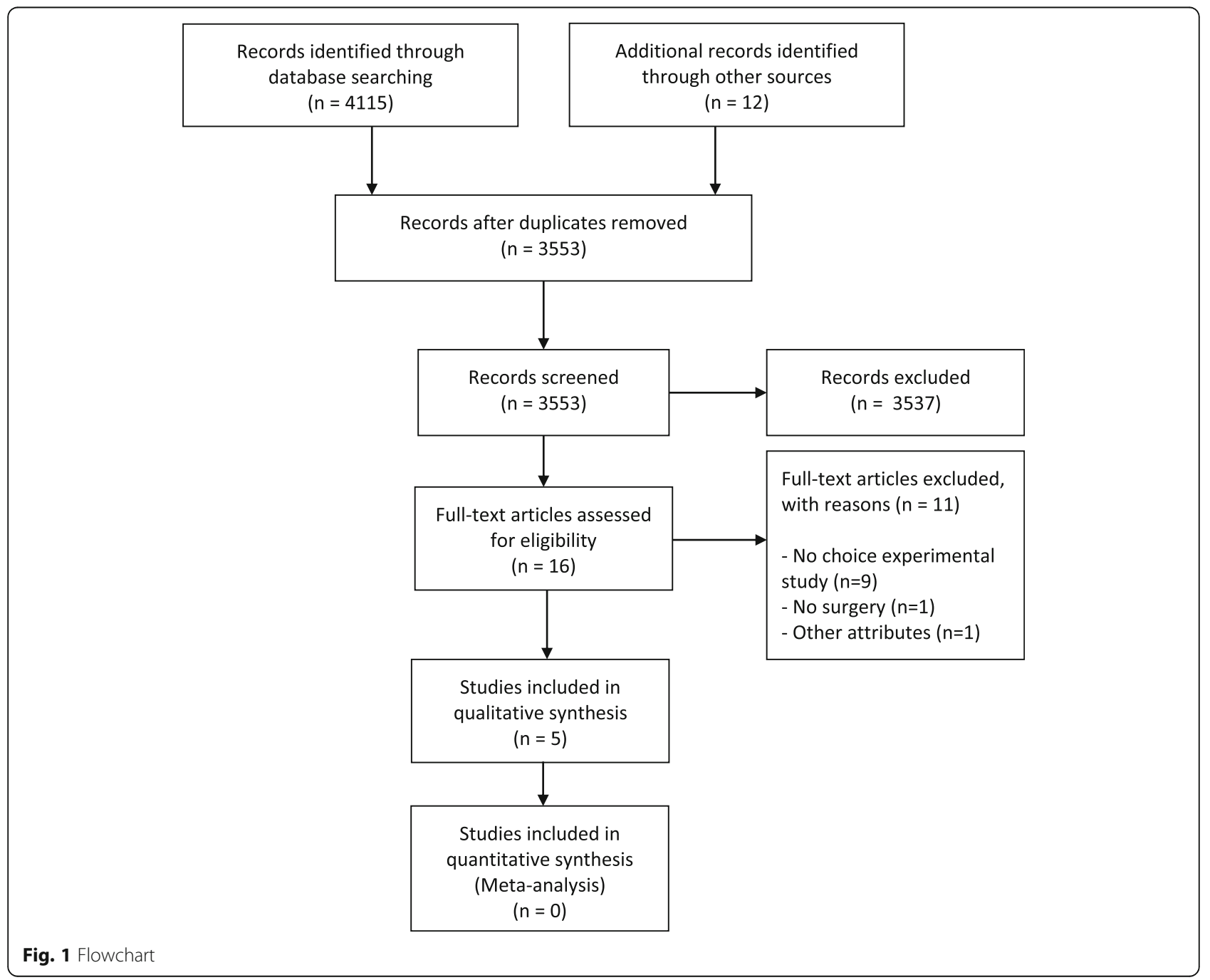


for the DCE. The distances to local and regional hospitals and the surgery-associated risks were presented either as text, graphs, and pictograms or as a combination of these risk presentations. Three studies performed two different DCEs (see Table 1) [13-15]. Shalowitz et al. used different risk presentations with different distances (50 miles with an increased survival benefit in the regional hospital, and 0-250 miles in nine increments with a fixed risk presented). In the study of Chang et al., in which parents were requested to make a decision for their children and for themselves, they were presented with two different distances for the farther hospital. In all studies, DCEs started with an equal risk in both the local and regional hospital. Burkamp et al. used two different outcomes (risk of 90-day mortality and of revision). In total, four studies used mortality risk. In three studies, mortality risk in the local hospital increased stepwise $[8,9,13]$ and in the study of Burkamp et al. [15], mortality risk and risk of revision in the regional hospital decreased. In the study of Shalowitz et al., the 5 -year survival rate increased in the regional hospital.

\section{Results of the DCEs of the individual studies}

Participants' preferences for local care varied between studies (see Table 2 and Additional file 3). In all studies, there were participants who, independently of a decreased mortality risk $[8,9,13,15]$, revision risk [15] or a higher survival benefit [14] in the regional hospital, adhered to the local hospital (3-10.7\%).In the study by Finlayson et al., nearly half of the participants (45\%) were willing to accept an additional risk to receive care locally [8]. They stratified by strength of preferences for local care, which showed that $21 \%$ of the participants accepted very high levels of additional risk (10 to $>50$ percentage points) rather than going to a regional hospital. Ten percent of participants chose local care, even if the mortality rate was $100 \%$.In contrast, Landau et al. found that the majority of participants (91\%) did not tolerate any additional risk to have surgery performed locally [9].In all studies, a proportion of participants (1-40\%) preferred the regional hospital for treatment although mortality rates were identical in both hospitals. Only in the studies by Finlayson et al. and Burkamp et al. (for the risk of revision), all participants (100\%) preferred to go to the local hospital when risks were equal. The study by Chang et al. showed that parents were more willing to travel for their children's care than for their own to keep mortality risk low, and that their decision depended on the travel distance. If the regional hospital distance was two driving-hours, participants more often chose the distant hospital compared to a 4-h driving distance.In Finlayson et al., 45\% of the participants preferred surgery the regional hospital after the risk at the local hospital was increased in the first step. In the study by
Chang et al., 36.9\% ( $2 \mathrm{~h}$-driving distances) respectively $48.5 \%$ (4-h drive) preferred to go to regional hospital for their child's surgery when the first increase of the risk in the local hospital was performed. In the first DCE of Shalowitz et al., where the distance to regional hospital was 50 miles, $29 \%$ of the participants chose the local hospital for treatment of ovarian cancer when survival was slightly higher in regional hospital (34\% versus $36 \%$ ). Burkamp et al. performed two DCEs, one for 90-day mortality risk and one for risk of revision. For the risk of mortality, 92 of 180 participants (51\%) chose the regional hospital for surgery when mortality risk decreased from 1 to $0.8 \%$ in regional hospital. When revision risk in regional hospital decreased by 2 percentage points $(10$ to $8 \%), 37 \%$ of the participants chose surgery in distant hospital.

\section{Associated factors}

Shalowitz et al. compared patient characteristics of those who were willing to travel an additional 50 miles for a $6 \%$ survival benefit and those who were not willing. Participants who were not employed ( $56 \%$ vs. $83 \%, p=0.05$ ) and who rated their own health as good to excellent ( $86 \%$ vs. $50 \%, p=0.04$ ) were more likely to travel. Landau et al. identified characteristics of patients who preferred regional care if risks in both hospitals were equal. A previous treatment in the regional hospital and presence of others living in the home were factors associated with acceptance to travel longer distances.

Finlayson et al. performed an ordinal logistic regression analysis and identified older age and fewer years of formal education with a higher acceptance of additional risk of surgery in a local hospital. Burkamp et al. performed a multivariate regression analysis to identify factors associated with patients' decisions. Surgery in the local hospital was preferred despite higher mortality risk by study participants who were recruited by registration office (in contrast to hospital recruitment), had a lower educational level, subjectively attached greater importance to hospital distance, or had friends or acquaintances with prior knee replacement surgery in that hospital. With regard to risk of revision, accessibility by public traffic and satisfaction with the number of visitors during a 10-day hospital stay were associated with preference for local care [15].

\section{Risk of bias of included studies}

Critical appraisal of the studies showed that three out of five studies $[8,9,13]$ had a serious risk of bias (RoB). The RoB for the studies by Shalowitz et al. and Burkamp et al. was rated as moderate $[14,15]$. In domain one, the selection of participants into the study, two studies showed a critical RoB $[8,13]$, one study had a serious [9] and another two a moderate $\operatorname{RoB}[14,15]$. In the second 


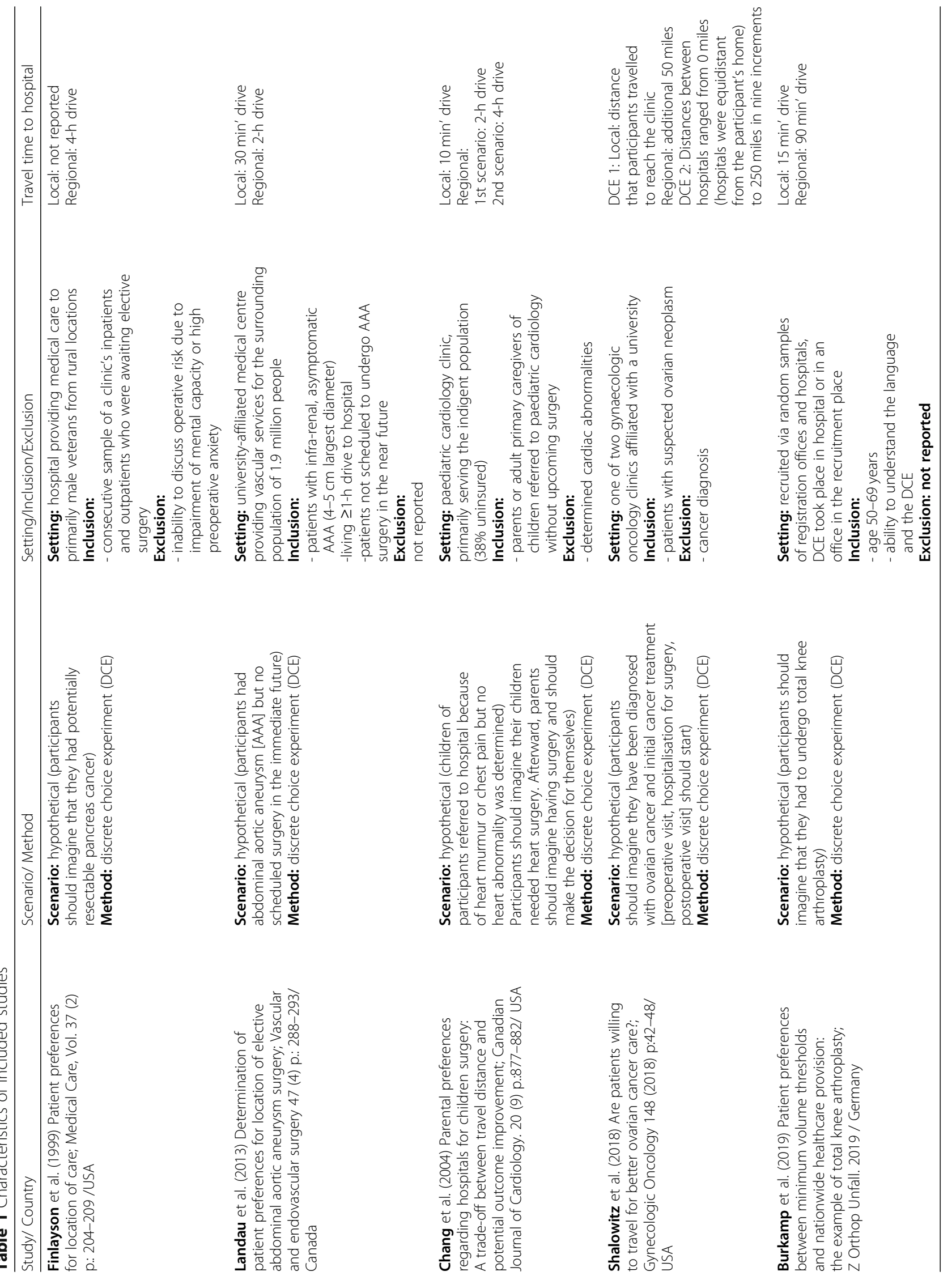


Table 2 Results of choice experiments

Study (number of patients) Outcome risk in local hospital Outcome in regional hospital

Proportions of participants remaining for surgery in local hospital N (\%)

\section{Outcome: mortality}

Finlayson $(N=100)^{a}$

$3 \%$

$6 \%$

$12 \%$

$18 \%$

$23 \%$

$100 \%$

Outcome: mortality

Landau $(N=67)$

\section{Outcome: mortality}

Changt $(N=103)$

Scenario 1

$$
3 \%
$$

$4 \%$

\section{$6 \%$}

$18 \%$

Scenario 2

$$
3 \%
$$$$
4 \%
$$$$
6 \%
$$$$
18 \%
$$

\section{Outcome: 5-year survival benefit}

Shalowitzt† ( $N=62)$

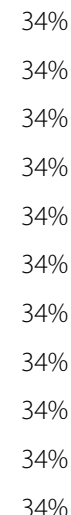

Outcome: mortality

Burkamp $(N=180)$

\section{$3 \%$ \\ $3 \%$ \\ $3 \%$ \\ $3 \%$ \\ $3 \%$ \\ $3 \%$}

$2 \%$

$2 \%$

$2 \%$

$2 \%$

$2 \%$

$3 \%$

$3 \%$

$3 \%$

$3 \%$

$3 \%$

$3 \%$

$3 \%$

$3 \%$

$34 \%$

$36 \%$

$38 \%$

$40 \%$

$42 \%$

$44 \%$

$46 \%$

$48 \%$

$50 \%$

$52 \%$

$54 \%$

$1 \%$

$0,8 \%$

$0,6 \%$

$0,4 \%$

$0,2 \%$

$0 \%$
100 (100\%)

45 (45\%)

23 (23\%)

$18(18 \%)$

17 (17\%)

$10(10 \%)$

$40\left(60 \%{ }^{b}\right)$

6 (9\%)

5 (7.5\%)

2 (3\%)

1 (1.5\%)

85 (82.5\%)

38 (36.9\%)

20 (19.4\%)

10 (9.7\%)

85 (82.5\%)

50 (48.5\%)

$23(22.3 \%)$

$11(10.7 \%)$

42 (68\%)

18 (29\%)

13 (21\%)

12 (19\%)

7 (11\%)

$6(10 \%)$

2 (3\%)

2 (3\%)

2 (3\%)

2 (3\%)

2 (3\%)

178 (99\%)

$92(51 \%)$

51 (28\%)

35 (19\%)

25 (14\%)

$11(6 \%)$ 
Table 2 Results of choice experiments (Continued)

\begin{tabular}{llll}
\hline Study (number of patients) & Outcome risk in local hospital & Outcome in regional hospital & $\begin{array}{l}\text { Proportions of participants remaining } \\
\text { for surgery in local hospital N (\%) }\end{array}$ \\
\hline Outcome: revision & $10 \%$ & $10 \%$ & $180(100 \%)$ \\
Burkamp $(N=180)$ & $10 \%$ & $8 \%$ & $67(37 \%)$ \\
& $10 \%$ & $6 \%$ & $25(14 \%)$ \\
$10 \%$ & $4 \%$ & $15(8 \%)$ \\
$10 \%$ & $2 \%$ & $10(6 \%)$ \\
& $10 \%$ & $0 \%$ & $6(3 \%)$ \\
\hline
\end{tabular}

In original study, there were differences in presentation of the results in figures and tables; we refer to the results presented in the result section of the study ${ }^{\mathrm{b}}$ Sixty-one participants who were not accepting any additional risk and number of participants accepting additional risk to keep surgery locally (number calculated)

t Only numbers of the decision of parents for their child's surgery reported in the original study

††Only DCE1 displayed

domain, which asked whether the instrument used for eliciting the relative importance of outcomes was valid and administered in an appropriate way, all but two studies $[14,15]$ showed a serious RoB. In the third domain (data analysis), the RoB was rated moderate in all studies. An overview of the RoB for the included studies is provided in Table 3.

\section{Discussion}

Our systematic review identified five studies that examined patients' preferences when trading off between surgery-associated risks and hospital distances. Both the surgery-associated risk and the distance seemed to have an influence on patients' decision making. In all studies, there was a general trend toward acceptance of greater travel distances if the surgical risk in the local hospital increased. However, in all studies, there was a fraction of participants who, despite a maximally increased risk, still preferred to undergo surgery in the local hospital (see Table 2). This leads to the assumption that, besides risk and distance, there might be other factors that have an influence on patients' decision-making process. One study identified unemployment and a rating of one's own health as good to excellent as characteristics associated with greater acceptance to travel [14]. Two studies performed regression analyses to identify participants' characteristics associated with the decision for the local hospital even if mortality risk there was higher $[8,15]$. Older age and fewer years of formal education were associated with preferences for local care in the study of Finlayson et al. [8]. Burkamp et al. also identified lower school-leaving qualifications as a factor associated with the willingness to accept higher mortality risks in the local hospital [15].

\section{Heterogeneity between the studies identified}

There was great variation in the characteristics of the included studies. The diseases used in the DCEs differed regarding severity and prognosis, which might have influenced choice behavior and willingness to travel. First, prognosis of diseases used in the scenarios varied greatly. One study used pancreatic cancer, which is one of the deadliest cancer types, with a 5-year relative survival rate of $6 \%$ for men and $8 \%$ for women in Europe [16]. Another study chose open-heart surgery (i.e., ventricular septal defect), which in contrast has a good prognosis [17].Second, despite the generally hypothetical nature of the DCEs there were differences between studies regarding the degree patients were affected with the scenario which likely influenced their ability to imagine being in the hypothetical situation. There were also differences regarding the study population. Although all studies used a hypothetical scenario, in one study [9], participants really suffered from the disease, which might have had an impact on the decision-making process. In one study, participants had the clinical suspicion of ovarian cancer and were referred to the clinic for clarification [14]. In contrast, in the study by Chang et al. [13], parents already knew that their children did not have heart abnormalities [13]. Therefore, decision making in the first study [14], where participants might receive a cancer diagnosis, might have been influenced by anxiety. In one study, patients had to make a decision between a local hospital and the regional hospital in which the study was conducted [9]. Third, in another study, participants were asked to imagine the local hospital was the hospital where the family in general receives care [13]. Research showed that a previously made personal experience of patients in a hospital was an influencing factor [5].

\section{Differences in risk presentation}

The kind of risk presented in the choice experiments differed between studies. One study used 5-year survival benefit [14], whereas the other studies used mortality risk $[8,9,13]$. Studies show that there are differences in 


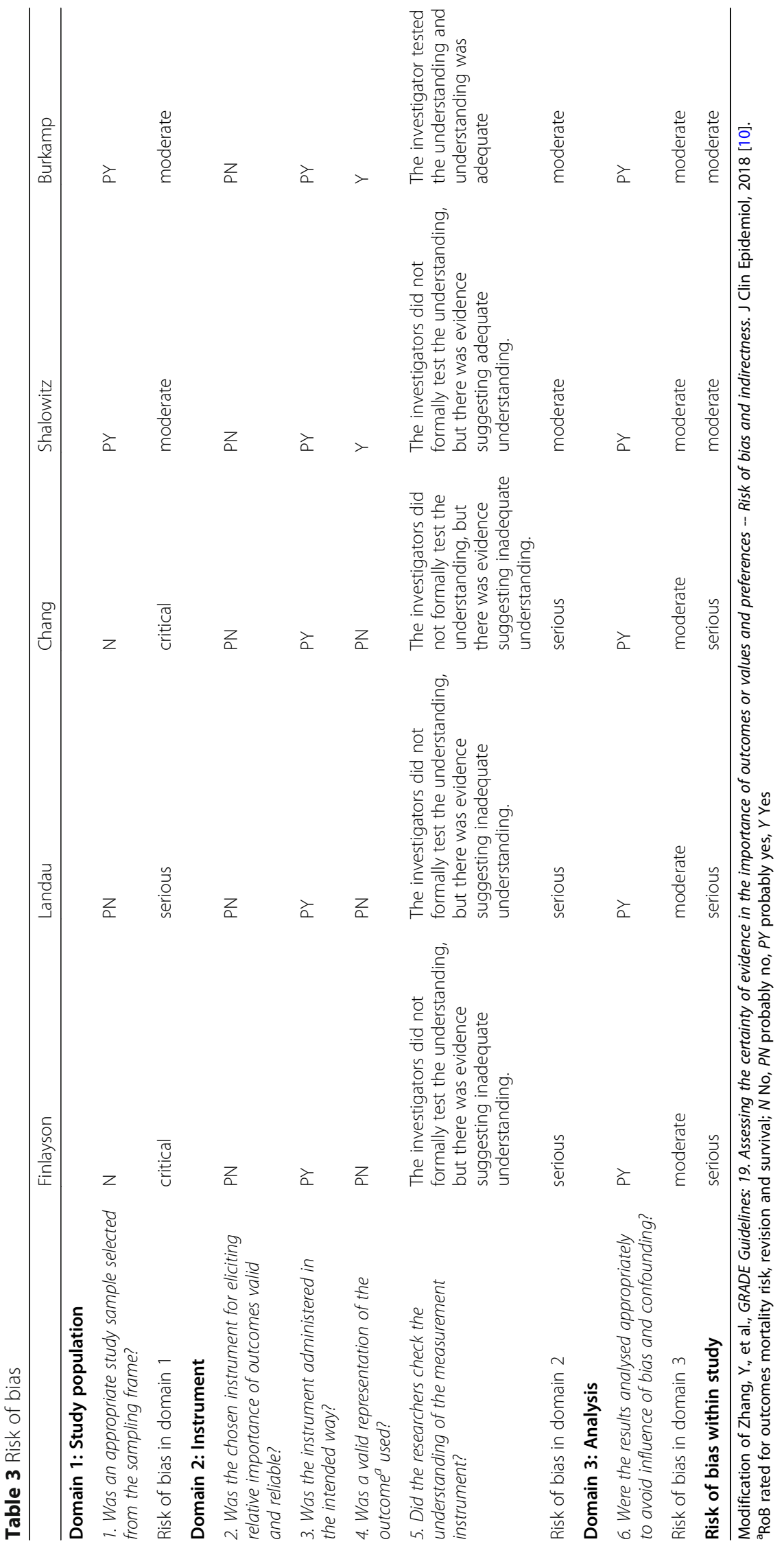


understanding risks and that the framing of the risk attribute (positive [survival] versus negative [mortality]) has an influence on decision-making behaviour in a DCE [18]. Negative risk framing leads to more riskseeking behaviour. Presentation of the risks of the choice experiments also differed, and only Shalowitz et al. and Burkamp et al. referred to evidence-based risk communication such as presentation of risks with both words and pictograms. Adequate understanding of the risks presented is an essential precondition to eliciting participants' preferences for local care, so it remains questionable whether the data presented in the studies reflects patients' actual preferences. Especially in Finlayson et al., when participants would stay in the local hospital even if they had a $100 \%$ mortality risk, the question arises whether they had properly understood the risks and consequences presented. In the majority of patients, health literacy is low. In addition, risk presentation was found to often be ambiguous [19]..

\section{Results of other studies}

Previous studies have identified additional factors influencing patients' choice of hospital for treatment.. The scoping review of Victoor et al. found that patients consider a variety of structural (e.g., availability), process (e.g., waiting time) and outcome (e.g., mortality rate) characteristics of providers [1]. Other studies in this field identified hospital and surgical reputation, surgical competency, personal experiences the patient had in a hospital and recommendations from relatives or outpatient personnel as influencing factors $[4,5]$. A study by Varkevisser et al. with empirical data on revealed preferences of patients with orthopaedic and neurosurgical surgery showed that extra travel time and good hospital waiting time performance affected the decisions to visit the hospital closest to patients' homes [11].

\section{Strengths and limitations}

To the best of our knowledge, this is the first systematic review on patients' preferences evaluating the trade-offs between distance to hospital and surgery-associated risks using choice experiments. Our study has several limitations. First, although we performed a systematic search and contacted study authors, we might have missed some relevant studies. The second limitation is that the included studies had a moderate to high risk of bias. To assess the RoB of the patients' preference studies we used a recently developed tool [12]. However, to the best of our knowledge it is not yet validated. Another limitation is that generalisability of the study findings is limited. All studies used a hypothetical scenario in which patients were asked to make a decision based on an imagined situation. The impact of the differences in choice behaviour between participants who were really suffering the disease and those who were not remains unclear. However, research shows that discrete choice experiments (DCEs) could predict real-world decisions [20]. Results of the studies might not be transferable to other health care systems and settings. In countries with relatively large geographical distances and low population density, such as Australia, the definition of reasonable distance to a hospital might differ compared to countries with higher population density [21]. Two of the authors (SB, DP) co-authored the study of Burkamp et al. [15]. Although we were aware of this conflict of interest, we could not completely exclude an influence on the quality rating of this study [22].

\section{Conclusions}

In all studies, there was a general trend toward the acceptance of greater travel distances if the surgical risk in the local hospital increased. The question of whether patients were willing to travel for a lower surgeryassociated risk could not be answered in a straightforward manner. The studies we identified showed that decision making does not only rely on rational information such as risk information and distance to hospital. Against the background of centralisation of clinical care and minimum surgery volumes, patient preferences regarding hospital choice are crucial to be considered by national policy decision makers. Our study shows a high need for more studies that consider patients' preferences and values. Future studies should investigate the influence of hospital distance on decision making, comparing diseases with different severity and prognosis, comparing specialised surgery with routine procedures. Risk presentation should rely on the newest risk communication research and a proper understanding that the choice experiments should be validated by pretesting the scenarios. Participants living in different settings (urban, suburban and rural) should be included to investigate the influence on willingness to travel.

\section{Supplementary information}

Supplementary information accompanies this paper at https://doi.org/10. 1186/s12889-020-8333-5.

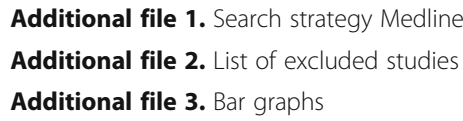

\section{Abbreviations}

AAA: Abdominal aortic aneurysm; DCE: Discrete choice experiment; GRADE: GRADING of Recommendations, Assessment, Development and Evaluation; PROSPERO: Prospective register of ongoing systematic reviews; RoB: Risk of Bias

Acknowledgements Not applicable. 


\section{Authors' contributions}

The development of the study concept and the systematic literature search were performed by DP. All authors participated in study selection and data collection. SB drafted the manuscript for submission. All authors were actively involved in the analysis, interpretation of results and the discussion section. All authors have approved the final version

\section{Funding}

No financial support for this study was provided. The funding agreement ensured the authors' independence in designing the study, interpreting the data, writing, and publishing the report.

\section{Availability of data and materials}

The datasets used and/or analyzed during the current study are available from the corresponding author on reasonable request.

\section{Ethics approval and consent to participate}

Not applicable.

\section{Consent for publication}

Not applicable.

\section{Competing interests}

The authors SB and DP are co-authors of the study of Burkamp et al. [15]. The authors declare that there is no other conflict of interest.

\section{Author details}

${ }^{1}$ Institute for Research in Operative Medicine, Faculty of Health, School of Medicine, University Witten/Herdecke, Ostmerheimer Str. 200, Building 38, D-51109 Cologne, Germany. ${ }^{2}$ Central Research Institute of Ambulatory Health Care in Germany (Zi), Salzufer 8, D-10587 Berlin, Germany

\section{Received: 4 May 2019 Accepted: 6 February 2020}

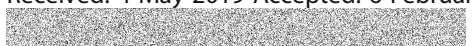

\section{References}

1. Victoor A, Delnoij DM, Friele RD, Rademakers JJ. Determinants of patient choice of healthcare providers: a scoping review. BMC Health Serv Res. 2012;12:272.

2. Magee H, Davis $L$, Coulter A. Public views on healthcare performance indicators and patient choice. J R Soc Med. 2003;96(7):338-42.

3. Victoor A, Rademakers J, Reitsma-van Rooijen M, de Jong J, Delnoij D, Friele $R$. The effect of the proximity of patients' nearest alternative hospital on their intention to search for information on hospital quality. J Health Serv Res Policy. 2014;19(1):4-11.

4. Yahanda AT, Lafaro KJ, Spolverato G, Pawlik TM. A systematic review of the factors that patients use to choose their surgeon. World J Surg. 2016;40(1): 45-55.

5. de Cruppe W, Geraedts M. Hospital choice in Germany from the patient's perspective: a cross-sectional study. BMC Health Serv Res. 2017;17(1):720.

6. Morche J, Mathes T, Pieper D. Relationship between surgeon volume and outcomes: a systematic review of systematic reviews. Syst Rev. 2016;5(1):204.

7. Pieper D, Mathes T, Neugebauer E, Eikermann M. State of evidence on the relationship between high-volume hospitals and outcomes in surgery: a systematic review of systematic reviews. J Am Coll Surg. 2013;216(5):101525.e18.

8. Finlayson SR, Birkmeyer JD, Tosteson AN, Nease RF Jr. Patient preferences for location of care: implications for regionalization. Med Care. 1999;37(2): 204-9.

9. Landau JH, Novick TV, Dubois L, Power AH, Harris JR, Derose G, et al. Determination of patient preference for location of elective abdominal aortic aneurysm surgery. Vasc Endovasc Surg. 2013;47(4):288-93.

10. Schwartz LM, Woloshin S, Birkmeyer JD. How do elderly patients decide where to go for major surgery? Telephone interview survey. BMJ (Clinical research ed). 2005;331(7520):821.

11. Varkevisser M, van der Geest SA. Why do patients bypass the nearest hospital? An empirical analysis for orthopaedic care and neurosurgery in the Netherlands. Eur J Health Econ. 2007:8(3):287-95.

12. Zhang Y, Alonso-Coello P, Guyatt GH, et al. GRADE Guidelines: 19. Assessing the certainty of evidence in the importance of outcomes or values and
preferences-Risk of bias and indirectness. J Clin Epidemiol. 2019;111:94-104. https://doi.org/10.1016/j.jclinepi.2018.01.013.

13. Chang RKR, Joyce JJ, Castillo J, Ceja J, Quan P, Klitzner TS. Parental preference regarding hospitals for children undergoing surgery: a trade-off between travel distance and potential outcome improvement. Can J Cardiol. 2004;20(9):877-82.

14. Shalowitz DI, Nivasch E, Burger RA, Schapira MM. Are patients willing to travel for better ovarian cancer care? Gynecol Oncol. 2018;148(1):42-8.

15. Burkamp J, Bühn S, Pieper D. Patient Preferences between Minimum Volume Thresholds and Nationwide Healthcare Provision: the Example of Total Knee Arthroplasty [published online ahead of print, 2019 Sep 16] [published correction appears in Z Orthop Unfall. 2020 Feb 03;:]. Z Orthop Unfall. 2019;10.1055/a-0965-7720. https://doi.org/10.1055/a-0965-7720.

16. De Angelis R, Sant M, Coleman MP, Francisci S, Baili P, Pierannunzio D, et al. Cancer survival in Europe 1999-2007 by country and age: results of EUROCARE-5-a population-based study. Lancet Oncol. 2014;15(1):23-34.

17. Nederend I, de Geus EJC, Blom NA, Ten Harkel ADJ. Long-term follow-up after ventricular septal defect repair in children: cardiac autonomic control, cardiac function and exercise capacity. Eur J Cardiothorac Surg. 2018;53(5): $1082-8$.

18. Veldwijk J, Essers BA, Lambooij MS, Dirksen CD, Smit HA, de Wit GA. Survival or mortality: does risk attribute framing influence decision-making behavior in a discrete choice experiment? Value Health. 2016;19(2):202-9.

19. Sorensen K, Pelikan JM, Rothlin F, Ganahl K, Slonska Z, Doyle G, et al. Health literacy in Europe: comparative results of the European health literacy survey (HLS-EU). Eur J Pub Health. 2015;25(6):1053-8.

20. Quaife M, Terris-Prestholt F, Di Tanna GL, Vickerman P. How well do discrete choice experiments predict health choices? A systematic review and metaanalysis of external validity. Eur J Health Econ. 2018;19(8):1053-66. https://doi.org/10.1007/s10198-018-0954-6.

21. Rechel B, Dzakula A, Duran A, Fattore G, Edwards N, Grignon M, et al. Hospitals in rural or remote areas: An exploratory review of policies in 8 high-income countries. Health policy (Amsterdam, Netherlands). 2016;120(7): 758-69.

22. Pieper D, Waltering A, Holstiege J, Buchter RB. Quality ratings of reviews in overviews: a comparison of reviews with and without dual (co-)authorship. Syst Rev. 2018;7(1):63.

\section{Publisher's Note}

Springer Nature remains neutral with regard to jurisdictional claims in published maps and institutional affiliations.

\section{Ready to submit your research? Choose BMC and benefit from:}

- fast, convenient online submission

- thorough peer review by experienced researchers in your field

- rapid publication on acceptance

- support for research data, including large and complex data types

- gold Open Access which fosters wider collaboration and increased citations

- maximum visibility for your research: over $100 \mathrm{M}$ website views per year

At BMC, research is always in progress.

Learn more biomedcentral.com/submissions 